\title{
HARMless: Transient Cortical and Sulcal Hyperintensity on Gadolinium-Enhanced FLAIR after Elective Endovascular Coiling of Intracranial Aneurysms
}

\author{
(D)S. Suthiphosuwan, (DC.C.-T. Hsu, and (D) A. Bharatha
}

\begin{abstract}
BACKGROUND AND PURPOSE: Cortical and sulcal hyperintensity on gadolinium-enhanced FLAIR has been increasingly recognized after iodinated contrast medium exposure during angiographic procedures. The goal of this study was to assess the relationship of cortical and sulcal hyperintensity on gadolinium-enhanced FLAIR against various variables in patients following elective endovascular treatment of intracranial aneurysms.
\end{abstract}

MATERIALS AND METHODS: We performed a retrospective review of 58 patients with 62 MR imaging studies performed within 72 hours following endovascular treatment of intracranial aneurysms. Patient demographics, aneurysm location, and vascular territory distribution of cortical and sulcal hyperintensity on gadolinium-enhanced FLAIR were documented. Analysis of cortical and sulcal hyperintensity on gadolinium-enhanced FLAIR with iodinated contrast medium volume, procedural duration, number of angiographic runs, and DWI lesions was performed.

RESULTS: Cortical and sulcal hyperintensity on gadolinium-enhanced FLAIR was found in 32/62 (51.61\%) post-endovascular treatment MR imaging studies, with complete resolution of findings in all patients on the available follow-up studies (27/27). Angiographic iodinated contrast medium injection and arterial anatomy matched the vascular distribution of cortical and sulcal hyperintensity on gadoliniumenhanced FLAIR. No significant association was found between cortical and sulcal hyperintensity on gadolinium-enhanced FLAIR with iodinated contrast medium volume ( $P=.56$ value) and the presence of DWI lesions $(P=.68)$. However, a significant association was found with procedural time $(P=.001)$ and the number of angiographic runs $(P=.019)$. No adverse clinical outcomes were documented.

CONCLUSIONS: Cortical and sulcal hyperintensity on gadolinium-enhanced FLAIR is a transient observation in the arterial territory exposed to iodinated contrast medium during endovascular treatment of intracranial aneurysms. Cortical and sulcal hyperintensity on gadolinium-enhanced FLAIR is significantly associated with procedural time, and the frequency of angiographic runs suggesting a potential technical influence on the breakdown of the BBB, but no reported adverse clinical outcome or association with both iodinated contrast medium volume and DWI lesions was found. Recognition of cortical and sulcal hyperintensity on gadolinium-enhanced FLAIR as a benign incidental finding is vital to avoid unnecessary investigation.

ABBREVIATIONS: ACA = anterior cerebral artery; CSHF = cortical and sulcal hyperintensity on gadolinium-enhanced FLAIR; EVT = endovascular treatment; Gd-FLAIR = gadolinium-enhanced FLAIR; HARM = hyperintense acute reperfusion marker; ICM = iodinated contrast medium; IQR = interquartile range; PCA = posterior cerebral artery; $\mathrm{VA}=$ vertebral artery

ortical and sulcal hyperintensity on MR imaging following a neuroangiographic procedure is an increasingly recognized clinicoradiologic phenomenon. Most data arises from the neuro-

Received August 28, 2017; accepted after revision December 15.

From the Division of Diagnostic and Interventional Neuroradiology, Department of Medical Imaging (S.S., C.C.-T.H., A.B.) and Division of Neurosurgery, Department of Surgery (A.B.), St. Michael's Hospital, University of Toronto, Toronto, Ontario,

Canada.

Please address correspondence to Suradech Suthiphosuwan, MD, FRCR, Department of Medical Imaging, St. Michael's Hospital, University of Toronto, 30 Bond St, Toronto, Ontario, M5B 1W8, Canada; e-mail: suradech164@gmail.com

http://dx.doi.org/10.3174/ajnr.A5561 radiology literature perhaps due to greater use of MR imaging following an angiographic procedure. ${ }^{1-7}$ The FLAIR sequence is known to be particularly sensitive for the detection of various pathologic processes, including subarachnoid hemorrhage, meningitis, stroke, leptomeningeal carcinomatosis, and hyperoxygenation. ${ }^{6,8-11}$ FLAIR can be acquired before or after gadolinium administration. On non-gadolinium-enhanced FLAIR, sulcal hyperintensity is the failure of nulling of normal CSF signal intensity. ${ }^{12}$ Gadolinium-enhanced FLAIR (Gd-FLAIR) has the added sensitivity of the T1 shortening effect after gadolinium injection and is more sensitive in the detection of leptomeningeal pathologies. ${ }^{12-14}$ 
Recently, cortical and sulcal hyperintensity on Gd-FLAIR (CSHF) has been observed following iodinated contrast medium (ICM) exposure, which has been hypothesized as a manifestation of blood-brain barrier impairment. ${ }^{7,15,16}$ Most interestingly, CSHF has been observed following neurovascular interventions with a striking dichotomy in its clinical significance. Following acute stroke interventions, CSHF has been shown to be an imaging marker of poor prognosis, with associated reperfusion, risk of hemorrhagic transformation, and poor clinical outcome. ${ }^{6,17-20}$ This phenomenon has been termed "hyperintense acute reperfusion marker (HARM)" and has been hypothesized as early disruption of the BBB in acute stroke. ${ }^{6,17,19,21,22}$ Similar findings were reported on the hemodynamic changes after carotid stent placement ${ }^{1,3,5,23}$ and after a cardiac bypass operation. ${ }^{24}$ Most interesting, CSHF may not be strictly associated with vascular pathologies or procedures because CSHF has more recently been described in normal aging, mild cognitive impairment, or dementia. CSHF ultimately may reflect a multifactorial process leading to focal loss of BBB integrity. ${ }^{25,26}$

Based on the limited evidence from the literature, there is speculation for a causative relationship between CSHF and ICM exposure. Complex pathophysiologic processes during endovascular procedures such as hypoperfusion, hypoxia, ischemia, and reperfusion injury or direct neurotoxicity of the contrast agent ${ }^{1-5,19,21,22}$ can result in transient injuries to the BBB.

At our institution, we routinely perform MR imaging with contrast-enhanced MRA in all our patients immediately post-endovascular treatment (EVT) of intracranial aneurysms as a baseline study for future comparison. We reviewed a large cohort of patients who underwent MR imaging within 72 hours after elective EVT of unruptured aneurysms and examined the associations of CSHF with ICM volume exposure, DWI lesions, duration of EVT, and number of angiographic runs.

\section{MATERIALS AND METHODS Patient Population}

We retrospectively reviewed 62 consecutive postcoiling MR imaging studies in 58 consecutive patients who underwent elective treatment or re-treatment of cerebral aneurysms from July 2015 to December 2016. The institutional review board approved the study and informed consent was waived.

\section{Endovascular Coiling}

Endovascular coiling was performed in our neuroangiography suite with Artis zee biplane neuroangiography equipment (Siemens, Erlangen, Germany). All patients underwent EVT under general anesthesia. Iohexol (Omnipaque, $300 \mathrm{mg} \mathrm{I/mL;} \mathrm{GE} \mathrm{Health-}$ care, Piscataway, New Jersey) low-osmolar ICM was consistently used across the cohort.

We routinely performed a right groin approach. Contrast angiographic runs were performed using a power injector with the rate of $4 \mathrm{~mL} / \mathrm{s}$ of $10-\mathrm{mL}$ total volume for internal carotid artery injection and $4 \mathrm{~mL} / \mathrm{s}$ of 8 - $\mathrm{mL}$ total volume for vertebral artery (VA) injection at both the beginning and the end of the coiling procedure. For the remainder of the procedure, the angiographic runs were performed by a hand-injection technique. After EVT, all patients were re-examined and assessed for any neurologic symptoms by neurointerventionalists before discharge.
We documented the side of major arteries where endovascular maneuvering and ICM injection were performed: ICA or VA, total volume of ICM, number of angiographic runs, duration of the EVT, and duration between the end of procedure and obtaining the MR imaging scan.

\section{MR Imaging Techniques}

All patients underwent MR imaging within 72 hours of completion of EVT of the aneurysm on a 1.5T MR imaging scanner (Achieva; Philips Healthcare, Best, the Netherlands) using a 6-channel head coil. All patients were already extubated and breathing room air at the time of the MRI (excluding hyperoxygenation as a cause for sulcal FLAIR hyperintensity). Our MR imaging protocol consisted of DWI, 3D-TOF MRA, contrast-enhanced MRA, and, last, Gd-FLAIR. Contrast-enhanced MRA was acquired after a dose of $20 \mathrm{~mL}$ of gadobenate dimeglumine (MultiHance, 529 mg/mL; Bracco Diagnostics, Princeton, New Jersey) was injected intravenously. The Gd-FLAIR acquisition used the following parameters: TR/TE/TI/flip angle $=11,000 \mathrm{~ms} /$ $140 \mathrm{~ms} / 2800 \mathrm{~ms} / 90^{\circ}$, slice thickness $=5 \mathrm{~mm}$, slice spacing $=6$ $\mathrm{mm}, \mathrm{NEX}=1$, matrix size $=240 \times 240$, FOV $=200 \mathrm{~mm}$. DWI was performed with TR/TE/flip angle $=3386.71 \mathrm{~ms} / 75 \mathrm{~ms} / 90^{\circ}$, $\mathrm{NEX}=4$, slice thickness $=5 \mathrm{~mm}$, slice spacing $=6 \mathrm{~mm}$, matrix $=$ $256 \times 256, \mathrm{FOV}=230 \mathrm{~mm}$, and b-values $=0$ and $1000 \mathrm{~s} / \mathrm{mm}^{2}$.

\section{Image Analysis}

All acquired images were transferred to our PACS, and patient identity on final images for interpretation was removed to facilitate blinded analysis. Two neuroradiologists (S.S. and C.C.-T.H.) independently evaluated each study with final agreement by consensus. Gd-FLAIR was evaluated for the presence of CSHF. Findings positive for CSHF were defined as cortical or sulcal signal intensity equal to or greater than a superficial cortical vein. The location of the CSHF was documented according to conventional arterial territory: right/left anterior cerebral artery (ACA), right/ left middle cerebral artery, right/left posterior cerebral artery (PCA), and right/left cerebellar hemispheres. DWI was evaluated for presence of lesions reflecting acute thromboembolic infarct, which was defined as a presence of high signal intensity focus of $\geq 15 \mathrm{~mm}$. The number of restricted diffusion foci were graded according to Kang et $\mathrm{al}^{27}$ and Kim et $\mathrm{al}^{28}: 0$ (none), I ( $<6$ foci of diffusion restriction), and II ( $\geq 6$ foci of diffusion restriction). When available, follow-up MR imaging studies 2-4 months following the index treatment were evaluated.

\section{Statistical Analysis}

Statistical analysis was performed using SPSS (Version 22.0; IBM, Armonk, New York). Continuous variables were reported as mean \pm SD or median with interquartile range (IQR) and were compared using the Student $t$ test or Mann-Whitney $U$ test as appropriate. The $\chi^{2}$ test or Fisher exact test was performed for categoric variables. Statistical significance was defined as $P<.05$.

\section{RESULTS}

\section{Patient Characteristics}

Sixty-two MR imaging studies in 58 patients (40 women and 18 men) with post-elective EVT were evaluated. The age ranged from 
Table 1: Patient demographics and aneurysms

\begin{tabular}{lc}
\hline Patient data & \\
No. & 58 \\
Age (mean) (range) (yr) & $57.17 \pm 10.94(33-78)$ \\
Sex (No.) (\%) & Female, $40(69 \%)$ \\
Endovascular coiling aneurysms (No.) & 62 \\
Target aneurysm locations (N=62) & \\
AComA (No.) (\%) & $10(16.13)$ \\
Right ICA (No.) (\%) & $12(19.35)$ \\
Left ICA (No.) (\%) & $8(12.9)$ \\
Right PcomA (No.) (\%) & $4(6.45)$ \\
Right MCA (No.) (\%) & $7(11.29)$ \\
Left MCA (No.) (\%) & $2(3.22)$ \\
BA (No.) (\%) & $17(27.42)$ \\
Left VA (No.) (\%) & $1(1.61)$ \\
Left PCA (No.) (\%) & $1(1.61)$ \\
Endovascular coiling techniques (N = 62) & \\
Routine coiling (No.) (\%) & $23(37.1 \%)$ \\
Balloon-assisted coiling (No.) (\%) & $29(46.77 \%)$ \\
Stent-assisted coiling (No.) (\%) & $6(9.68 \%)$ \\
Pipeline flow diverter and coiling (No.) (\%) & $3(4.8 \%)$ \\
Balloon- and stent-assisted coiling (No.) (\%) & $1(1.61 \%)$ \\
No. of coils (median) (range) & $5(1-32)$
\end{tabular}

Note:-AcomA indicates anterior communicating artery; PcomA, posterior communicating artery; BA, basilar artery.

33 to 78 years with a mean of 57 years. None of these patients had SAH before or during the elective EVT. Various endovascular techniques were used for treatment of aneurysms or re-treatment for recanalized aneurysms. Of 62 EVTs, the location of the target coiling of the aneurysms was as follows: anterior communicating artery $(n=10)$, right ICA $(n=12)$, left ICA $(n=8)$, right posterior communicating artery $(n=4)$, right MCA $(n=7)$, left MCA $(n=2)$, basilar artery $(n=17)$, left VA $(n=1)$, and left PCA $(n=$ 1). Of the 62 EVTs, 30 studies assessed the target aneurysm via the right ICA as a major angiographic run with ICM. Thirteen studies were assessed via the left ICA. Six studies were assessed via the right VA. Thirteen studies were assessed using the left VA. Detailed data on patient demographics, aneurysm characteristics, and EVT are shown in Table 1.

\section{CSHF: Incidence and Location}

Thirty-two MR imaging studies (51.61\%) were positive for CSHF, and $30(48.39 \%)$ were negative. No significant difference between age and sex of the patients $(P=.77$ and $P=.12$, respectively) was observed between groups positive and negative for CSHF. Of 32 MR imaging studies positive for CSHF, the CSHF was found in the right ACA territory (Fig 1A) (15/32, 46.87\%), right MCA territory (Fig $2 B)(18 / 32,56.25 \%)$, left ACA territory (Fig 1C) (4/32, 12.5\%), left MCA territory (Fig 1C) (4/32, 12.5\%), right PCA territory (Fig $2 B)(6 / 32,18.75 \%)$, left PCA territory $(4 / 32,12.5 \%)$, and left cerebellar hemispheric territory (2/32, 6.25\%) (Fig 1E).

In the group positive for CSHF, 7 CT scans of the brain obtained immediately after EVT showed no evidence of sulcal hyperdensity. Twenty-seven follow-up MR imaging studies were performed within 2-4 months, and all demonstrated complete resolution of CSHF.

\section{CSHF and Site of Angiographic Injection}

For the right ICA injection $(n=30)$, CSHF was found in the right ACA territory (13/30, 43.33\%) (Fig 1A), right MCA (Fig 2B) (18/
$30,60 \%)$, left ACA (1/30, 3.33\%), and right PCA (Fig 2B) (1/30, $3.33 \%)$. The left ICA injection $(n=13)$ showed that CSHF was found in the left ACA (Fig 1C) (3/13, 23.07\%), left MCA (Fig 1C) $(4 / 13,30.77 \%)$, and right ACA $(2 / 13,15.38 \%)$. The right VA injection $(n=6)$ demonstrated CSHF on the right PCA $(1 / 6$, $16.67 \%)$ and left PCA $(1 / 6,16.67 \%)$. The left VA injection $(n=$ 13 ) showed right PCA CSHF $(4 / 13,30.77 \%)$, left PCA CSHF $(3 / 13,23.08 \%)$, and left cerebellum CSHF $(2 / 13,15.38 \%)$ (Fig $1 E)$.

\section{CSHF and ICM Volume}

No significant difference in the ICM volume $(P=.588)$ was found between the 2 groups. The mean volume of the ICM in the group negative for CSHF was $453.33 \mathrm{~mL}$ (range, $200-800 \mathrm{~mL}$ ), and in the group positive for CSHF, $431.25 \mathrm{~mL}$ (range, $200-800 \mathrm{~mL}$ ). Similarly, no significant difference in the contrast volume per body weight was found between the 2 groups $(P=.566)$. The mean volume of contrast per body weight in the group positive for CSHF was $6.15 \mathrm{~mL} / \mathrm{kg}$ (range, $1.92-11.54 \mathrm{~mL} / \mathrm{kg}$ ), and in the group negative for CSHF, $6.53 \mathrm{~mL} / \mathrm{kg}$ (range, 3.04-12.35 $\mathrm{mL} / \mathrm{kg}$ ) (Table 2).

\section{CSHF and Procedural Time}

A significant difference was found in the procedural time between the 2 groups $(P=.001)$. The group positive for CSHF had a longer procedural time, with a median of 2.21 hours (range, 1.07-5.13 hours) compared with the group negative for CSHF, with a median of 1.39 hours (range, $0.7-4.12$ hours) (Table 2).

\section{CSHF and Number of Angiographic Runs}

A significantly higher number of angiographic runs was documented in the group positive for $\operatorname{CSHF}(P=.019)$, with a median number of 18 runs (range, 7-30 runs) and a median number of angiographic runs of 13 (range, 7-29 runs) in the group negative for CSHF group (Table 2).

\section{CSHF and Time from Coiling to MR Imaging}

No significant differences were found between the groups positive and negative for CSHF $(P=.87)$. Overall the time from completion of EVT to MR imaging in all patients ranged from 3.93-64.27 hours (median, 16.16 hours). In the group positive for CSHF, the time to MR imaging ranged from 3.93 to 64.27 hours (median, 16.08 hours). In the group negative for CSHF, it ranged from 6.6 to 46.17 hours (median, 16.16 hours) (Table 2).

\section{CSHF and DWI Lesions}

There was no significant correlation between grading of DWI lesions and the presence of CSHF $(P=.68)$. Twenty-two MR imaging studies (35.5\%) showed no DWI lesions (grade 0): 13 studies positive for CSHF versus 9 studies negative for CSHF. Twenty-five MR imaging studies (40.3\%) had grade I DWI lesions: 12 studies positive for CSHF versus 13 studies negative for CSHF. Finally, 15 MR imaging studies (24.2\%) showed grade II DWI lesions: 7 in the positive for CSHF group versus 8 in the negative for CSHF group (Table 2). No large-volume confluent/territorial infarct was encountered in our series. 

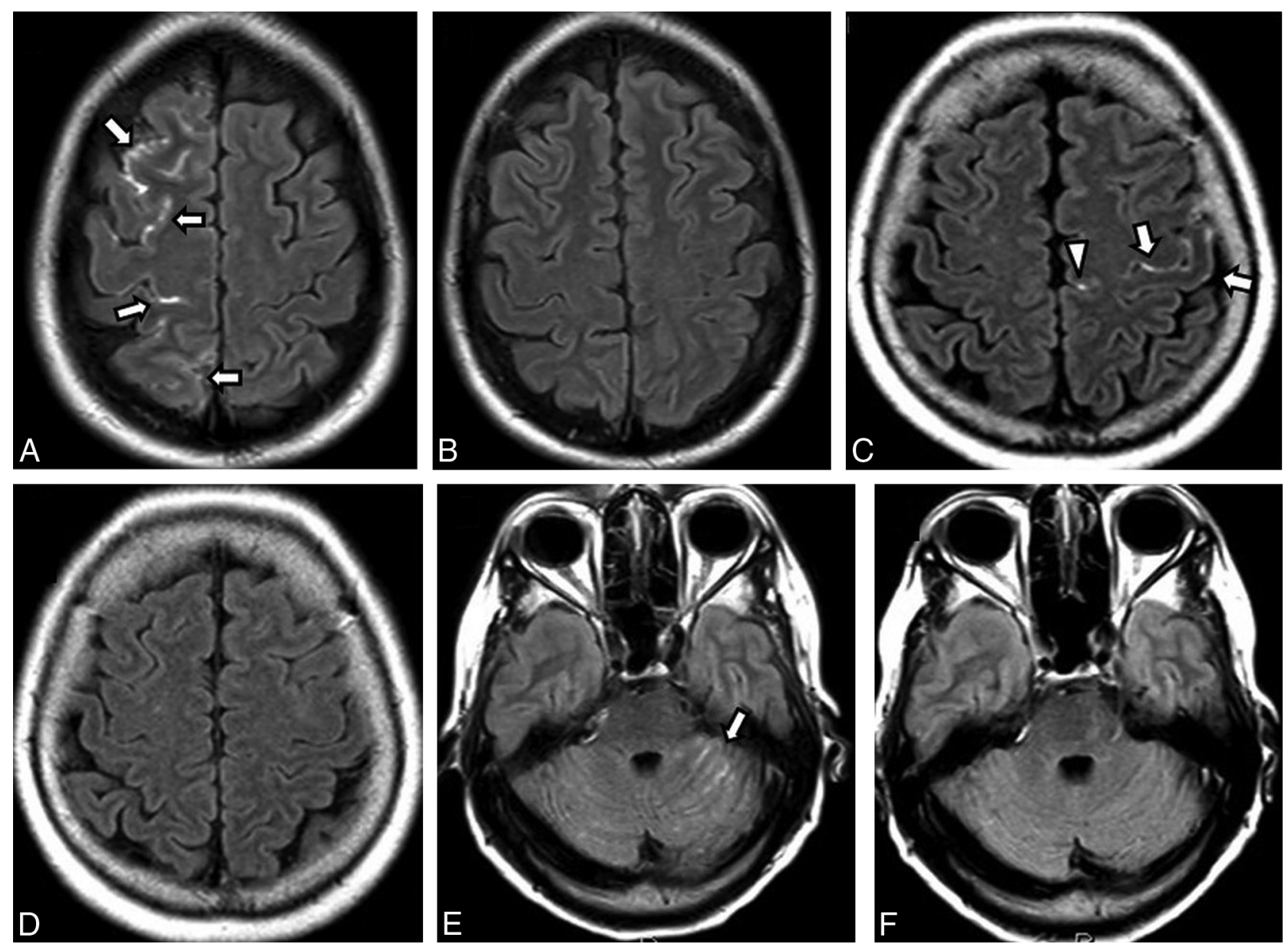

FIG 1. Different patterns of cortical and sulcal hyperintensity on gadolinium-enhanced FLAIR in different patients. $A$ and $B$, A 49-year-old woman with a postcoiling unruptured anterior communicating artery aneurysm via a right internal carotid artery approach. $A$, Postcoiling gadolinium-enhanced FLAIR shows CSHF along the right anterior cerebral artery territory (white arrows). $B$, Two-month follow-up Gd-FLAIR shows no residual abnormality. $C$ and $D, A$ 64-year-old woman postcoiling of a left paraclinoid ICA aneurysm via a left ICA approach. $C$, Postcoiling Gd-FLAIR shows CSHF along the left middle cerebral artery territory (white arrows) and left ACA territory (white arrowhead). $D$, Two-month follow-up Gd-FLAIR shows resolution of the abnormality. $E$ and $F$, A 61-year-old woman with a postcoiling basilar tip artery aneurysm via a left vertebral artery approach. E, Postprocedural Gd-FLAIR shows CSHF along the left cerebellar fissures (white arrow). F, Two-month follow-up Gd-FLAIR shows no residual abnormality.
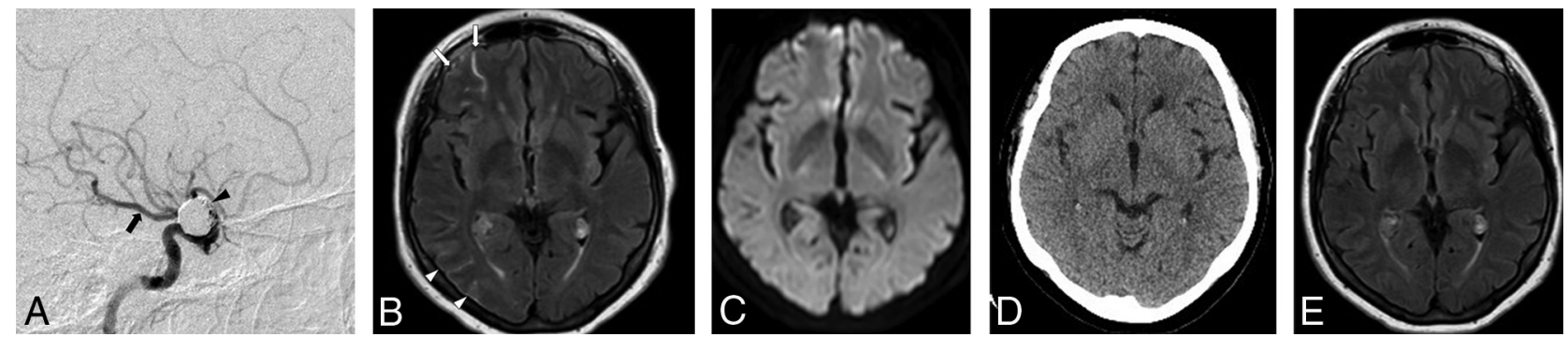

FIG 2. A, Right internal carotid artery angiographic approach for coiling of a right ophthalmic ICA aneurysm (black arrowhead) in a 71-year-old woman using $7.78 \mathrm{~mL} / \mathrm{kg}$ of iodinated contrast volume and a procedural time of 4 hours. Note the presence of fetal origin of the right posterior cerebral artery (black arrow). B, Gadolinium-enhanced FLAIR performed at 12 hours after endovascular coiling shows cortical and sulcal hyperintensity along the right middle cerebral artery territory (white arrows) and the right PCA territory (white arrowheads). C, Corresponding DWI shows no diffusion restriction. $D$, A follow-up unenhanced CT scan obtained on the same date shows no evidence of subarachnoid hemorrhage. E, Two-month follow-up Gd-FLAIR shows no residual abnormality.

\section{DISCUSSION}

CSHF is a radiologic phenomenon frequently observed in patients following elective EVT of unruptured aneurysms with an incidence of $51.61 \%$ (32/62 MR imaging studies). This study is the first of its kind to examine CSHF on MR imaging in a large asymp- tomatic cohort of patients following endovascular coiling of intracranial aneurysms, to our knowledge. The phenomenon of contrast extravasation into the subarachnoid space had been described on CT immediately post-EVT of intracranial aneurysms with a reported incidence of up to $23 \%-49 \%{ }^{29,30}$; however, in our 


\begin{tabular}{|c|c|c|c|c|}
\hline Factors & $\begin{array}{l}\text { Findings Negative } \\
\text { for CSHF }(n=30)\end{array}$ & $\begin{array}{l}\text { Findings Positive } \\
\text { for CSHF }(n=32)\end{array}$ & $\begin{array}{c}\text { Total } \\
(N=62)\end{array}$ & $\begin{array}{c}P \\
\text { Value }\end{array}$ \\
\hline Contrast volume (mean) (mL) & $453.33 \pm 156.98$ & $431.25 \pm 161.52$ & $441.93 \pm 158.42$ & .588 \\
\hline Contrast volume per weight (mean) (mL/kg) & $6.53 \pm 2.39$ & $6.15 \pm 2.56$ & $6.33 \pm 2.46$ & .556 \\
\hline Duration of endovascular coiling procedure (median) (IQR) (hr) & $1.39(0.95-2.20)$ & $2.21(1.63-3.26)$ & $1.89(1.26-2.66)$ & .001 \\
\hline Time from coiling to MRI (median) (IQR) (hr) & $16.16(11.99-22.51)$ & $16.08(10.03-22.61)$ & $16.16(11.56-22.125)$ & .871 \\
\hline No. of angiographic runs (median) (IQR) & $13(10-17)$ & $18(12-22)$ & $14(12-20)$ & .019 \\
\hline \multicolumn{5}{|l|}{ DWI grading (No.) (\%) } \\
\hline 0 & 9 & 13 & $22(35.5 \%)$ & .68 \\
\hline । & 13 & 12 & $25(40.3 \%)$ & \\
\hline II & 8 & 7 & 15 (20.2\%) & \\
\hline
\end{tabular}

series, subsequent CT did not show sulcal hyperdensity when performed. In addition, CSHFs were in the peripheral/cortical sulci far from the basal cisterns and hence unlikely to represent SAH or aneurysm perforation. CSHF appears to be transient, with resolution of the finding on available follow-up MR imaging. Reassuringly, no symptom or adverse clinical outcome was attributed to the observation of CSHF. The incidence of CSHF in our cohort is similar to that reported in patients following ischemic stroke therapy, which ranges from $33 \%$ to $45 \%{ }^{17,19,24}$ However, the clinical significance of CSHF may be dependent on the clinical context. In the setting of acute stroke, CSHFs are known to be predictors of hemorrhagic transformation and poor clinical outcome and are termed HARMs for this particular reason. ${ }^{6,17-19,21,31}$ We hypothesized that CSHF may be a radiologic manifestation of inherent vulnerability of the cerebral cortex, causing transient impairment of the BBB, allowing extravasation of gadolinium from the intravascular space into the subarachnoid space.

CSHFs in our study were observed in the arterial territories exposed to the ICM during the angiographic procedure (Figs 1 and 2). ${ }^{1,3,5,23}$ In addition, when variant arterial anatomy is present, CSHF could also be seen in the vascular territory perfused by the variant vessel (Fig 2).

Our study failed to demonstrate a significant correlation between total ICM volume and CSHF. Two previous studies ${ }^{29,30}$ showed a correlation between sulcal hyperdensity seen on CT and ICM volume in patients post-EVT of intracranial aneurysms. A small selective subset of patients from the study by Ozturk et $\mathrm{al}^{30}$ also underwent further MR imaging evaluation after detection of sulcal hyperdensity on CT, but the MR imaging examination findings were unremarkable. In our study, we only included patients with unruptured intracranial aneurysms, hence eliminating prior subarachnoid hemorrhage as a potential confounder, whereas both prior studies included ruptured intracranial aneurysms. More important, Gd-FLAIR is far more sensitive than CT in the detection of disease of the subarachnoid space.

A small case series by Wilkinson et $\mathrm{al}^{1}$ reported unilateral leptomeningeal enhancement on Gd-FLAIR in all their patients $(n=$ 12) after carotid stent placement. Ogami et $\mathrm{al}^{3}$ also reported a high incidence of $57 \%$ of this observation in a small case series $(n=21)$ post-carotid stent placement. The central hypothesis proposed was BBB breakdown after reperfusion or hemodynamic disturbance. In both studies, the dose of the ICM was not reported.

Contrast-induced encephalopathy is a rare complication reported after ICM exposure following diagnostic or interventional angiography. Neuroimaging findings of contrast-induced en- cephalopathy usually show unilateral or bilateral cerebral hemispheric edema and leptomeningeal enhancement, and the affected brain parenchyma often demonstrates signal abnormality on both FLAIR and DWI. ${ }^{7,15,16}$ On the basis of case reports and the small number of case series of contrast-induced encephalopathy, a speculative ICM volume threshold had been reported, which ranged from 75 to $1500 \mathrm{~mL} .^{15,16,32-34}$ The average contrast volume used in our study was $441.94 \mathrm{~mL}$, which is not significantly different from the reported ranges in the literature; however, no acute encephalopathy was observed in our series.

Vulnerability or injury to the brain parenchyma can weaken the BBB, making it more susceptible to permeability impairment. A measurable surrogate of parenchymal injury is DWI lesions burden indicating acute thromboembolic infarcts. The incidence of MR imaging studies with DWI lesions following endovascular coiling of unruptured aneurysms ranges between $37.3 \%$ and $54.5 \%,{ }^{27,28,35}$ which is similar to our reported incidence of $64.5 \%$. Our study did not show a significant correlation between grades of DWI abnormality and CSHF. Our result is consistent with the findings of Merino et $\mathrm{al},{ }^{24}$ which showed no significant correlation between DWI lesions and sulcal enhancement on Gd-FLAIR following cardiac bypass surgery.

Variation in angioarchitecture of the aneurysm means that a multitude of endovascular techniques are at the disposal of neurointerventionalists. A simple aneurysm may only necessitate deployment of coils, whereas larger and complex aneurysms may require assistance of a balloon microcatheter or stent. Differences in endovascular devices and techniques can have different hemodynamic impacts on the brain parenchyma but are difficult to quantify and were not addressed in this paper. Of the 4 major variables analyzed, procedural time and number of angiographic runs showed a significant correlation with CSHF. We hypothesize that perhaps it is not the total ICM volume or number of DWI lesions that correlates with weakened BBB but the frequency of contrast delivery and procedural catheter dwell time that predispose to the development of CSHF. Contrary to the HARM phenomenon following EVT of ischemic stroke, CSHF is a benign transient finding.

This study has several limitations including the retrospective nature of data collection. In addition, the MR imaging protocol does not include precontrast FLAIR; hence, we cannot unequivocally distinguish intrinsic FLAIR changes from gadolinium leak. Sometimes, we were unable to directly visualize the location of the catheter tip during angiographic runs due to technical factors, including the tip lying outside the FOV or inadequate visualiza- 
tion due to poor magnification in which case we used the previous run and opacified branches to infer the location. The total ICM volume was recorded as the total number of vials used. This is a overestimation of the actual ICM injected because we anticipated contrast spillage and contrast retained within the syringes or injectors. The total ICM volume showed no significant association with CSHF; however, this is unlikely to be reflective of the local ICM volume exposure. Local concentration of ICM volume maybe a more important variable to analyze than total ICM volume, but such data would be difficult to obtain retrospectively. Last, there may be a difference in individual sensitivity to ICM.

\section{CONCLUSIONS}

CSHF is a transient benign finding commonly seen following elective aneurysm coiling, which is observed in the vascular territory exposed to the ICM. There are statistically significant associations of CSHF with procedural time and the frequency of angiographic injections, but there is no direct relationship with total ICM volume or DWI lesions. It is vital for radiologists and clinicians to recognize CSHF because misdiagnosis can lead to unnecessary clinical concern or investigation.

\section{REFERENCES}

1. Wilkinson ID, Griffiths PD, Hoggard N, et al. Unilateral leptomeningeal enhancement after carotid stent insertion detected by magnetic resonance imaging. Stroke 2000;31:848-51 CrossRef Medline

2. Vangosa AB, Tortora D, Modestino F, et al. Reversible and asymptomatic gyral and subarachnoid contrast enhancement after carotid stenting. Neuroradiol J 2015;28:184-86 CrossRef Medline

3. Ogami R, Nakahara T, Hamasaki O, et al. Cerebrospinal fluid enhancement on fluid attenuated inversion recovery images after carotid artery stenting with neuroprotective balloon occlusions: hemodynamic instability and blood-brain barrier disruption. Cardiovasc Intervent Radiol 2011;34:936-41 CrossRef Medline

4. Fukushima Y, Nakahara I, Ohta T, et al. Rare complication characterized by late-onset transient neurological symptoms without hyperperfusion after carotid artery stenting: a report of three cases. Interv Neuroradiol 2015;21:72-79 CrossRef Medline

5. Cho HJ, Kim YJ, Lee JH, et al. Post-carotid stenting reperfusion injury with blood-brain barrier disruption on gadolinium-enhanced FLAIR MRI. BMC Neurol 2014;14:178 CrossRef Medline

6. Kim EY, Kim SS, Na DG, et al. Sulcal hyperintensity on fluid-attenuated inversion recovery imaging in acute ischemic stroke patients treated with intra-arterial thrombolysis: iodinated contrast media as its possible cause and the association with hemorrhagic transformation. J Comput Assist Tomogr 2005;29:264-69 CrossRef Medline

7. Chen K, Obaid S, Guillon-Létourneau L, et al. Iodinated contrast encephalopathy. Can J Neurol Sci 2017;44:432-33 CrossRef Medline

8. Singh SK, Agris JM, Leeds NE, et al. Intracranial leptomeningeal metastases: comparison of depiction at FLAIR and contrast-enhanced MR imaging. Radiology 2000;217:50-53 CrossRef Medline

9. Taoka T, Yuh WT, White ML, et al. Sulcal hyperintensity on fluidattenuated inversion recovery MR images in patients without apparent cerebrospinal fluid abnormality. AJR Am J Roentgenol 2001; 176:519-24 CrossRef Medline

10. Frigon C, Shaw DW, Heckbert SR, et al. Supplemental oxygen causes increased signal intensity in subarachnoid cerebrospinal fluid on brain FLAIR MR images obtained in children during general anesthesia. Radiology 2004;233:51-55 CrossRef Medline

11. Iancu-Gontard D, Oppenheim C, Touze E, et al. Evaluation of hyperintense vessels on FLAIR MRI for the diagnosis of multiple intracerebral arterial stenoses. Stroke 2003;34:1886-91 CrossRef Medline

12. Morris JM, Miller GM. Increased signal in the subarachnoid space on fluid-attenuated inversion recovery imaging associated with the clearance dynamics of gadolinium chelate: a potential diagnostic pitfall. AJNR Am J Neuroradiol 2007;28:1964-67 CrossRef Medline

13. Mamourian AC, Hoopes PJ, Lewis LD. Visualization of intravenously administered contrast material in the CSF on fluid-attenuated inversion-recovery MR images: an in vitro and animalmodel investigation. AJNR Am J Neuroradiol 2000;21:105-11 Medline

14. Bozzao A, Floris R, Fasoli F, et al. Cerebrospinal fluid changes after intravenous injection of gadolinium chelate: assessment by FLAIR MR imaging. Eur Radiol 2003;13:592-97 Medline

15. Guimaraens L, Vivas E, Fonnegra A, et al. Transient encephalopathy from angiographic contrast: a rare complication in neurointerventional procedures. Cardiovasc Intervent Radiol 2010;33:383-88 CrossRef Medline

16. Leong S, Fanning NF. Persistent neurological deficit from iodinated contrast encephalopathy following intracranial aneurysm coiling: a case report and review of the literature. Interv Neuroradiol 2012; 18:33-41 CrossRef Medline

17. Renú A, Laredo C, Lopez-Rueda A, et al. Vessel wall enhancement and blood-cerebrospinal fluid barrier disruption after mechanical thrombectomy in acute ischemic stroke. Stroke 2017;48:651-57 CrossRef Medline

18. Latour LL, Kang DW, Ezzeddine MA, et al. Early blood-brain barrier disruption in human focal brain ischemia. Ann Neurol 2004;56: 468-77 CrossRef Medline

19. Warach S, Latour LL. Evidence of reperfusion injury, exacerbated by thrombolytic therapy, in human focal brain ischemia using a novel imaging marker of early blood-brain barrier disruption. Stroke 2004;35:2659-61 CrossRef Medline

20. Mathews VP, Caldemeyer KS, Lowe MJ, et al. Brain: gadoliniumenhanced fast fluid-attenuated inversion-recovery MR imaging. Radiology 1999;211:257-63 CrossRef Medline

21. Lee KM, Kim JH, Kim E, et al. Early stage of hyperintense acute reperfusion marker on contrast-enhanced FLAIR images in patients with acute stroke. AJR Am J Roentgenol 2016;206:1272-75 CrossRef Medline

22. Köhrmann M, Struffert T, Frenzel T, et al. The hyperintense acute reperfusion marker on fluid-attenuated inversion recovery magnetic resonance imaging is caused by gadolinium in the cerebrospinal fluid. Stroke 2012;43:259-61 CrossRef Medline

23. Liu JJ, Dahlin BC, Waldau B. Contrast encephalopathy after coiling in the setting of obstructive sleep apnoea. BMJ Case Rep 2015;2015 pii: bcr2014207503 CrossRef Medline

24. Merino JG, Latour LL, Tso A, et al. Blood-brain barrier disruption after cardiac surgery. AJNR Am J Neuroradiol 2013;34:518-23 CrossRef Medline

25. Zenaro E, Piacentino G, Constantin G. The blood-brain barrier in Alzheimer's disease. Neurobiol Dis 2017;107:41-56 CrossRef Medline

26. Freeze WM, Schnerr RS, Palm WM, et al. Pericortical enhancement on delayed postgadolinium fluid-attenuated inversion recovery images in normal aging, mild cognitive impairment, and Alzheimer disease. AJNR Am J Neuroradiol 2017;38:1742-47 CrossRef Medline

27. Kang DH, Kim BM, Kim DJ, et al. MR-DWI-positive lesions and symptomatic ischemic complications after coiling of unruptured intracranial aneurysms. Stroke 2013;44:789-91 CrossRef Medline

28. Kim B, Kim K, Jeon P, et al. Thromboembolic complications in patients with clopidogrel resistance after coil embolization for unruptured intracranial aneurysms. AJNR Am J Neuroradiol 2014;35: 1786-92 CrossRef Medline

29. Brisman JL, Jilani M, McKinney JS. Contrast enhancement hyperdensity after endovascular coiling of intracranial aneurysms. AJNR Am J Neuroradiol 2008;29:588-93 CrossRef Medline

30. Ozturk A, Saatci I, Pamuk AG, et al. Focal increased cortical density in 
immediate postembolization CT scans of patients with intracranial aneurysms. AJNR Am J Neuroradiol 2006;27:1866-75 Medline

31. Lee H, Kim E, Lee KM, et al. Clinical implications of sulcal enhancement on postcontrast fluid attenuated inversion recovery images in patients with acute stroke symptoms. Korean J Radiol 2015;16: 906-13 CrossRef Medline

32. Spina R, Simon N, Markus R, et al. Contrast-induced encephalopathy following cardiac catheterization. Catheter Cardiovasc Interv 2017;90:257-68 CrossRef Medline
33. Dangas G, Monsein LH, Laureno R, et al. Transient contrast encephalopathy after carotid artery stenting. $J$ Endovasc Ther 2001;8: 111-13 Medline

34. Menna D, Capoccia L, Rizzo AR, et al. An atypical case of contrastinduced encephalopathy after carotid artery stenting. Vascular 2013;21:109-12 CrossRef Medline

35. Park JC, Lee DH, Kim JK, et al. Microembolism after endovascular coiling of unruptured cerebral aneurysms: incidence and risk factors. J Neurosurg 2016;124:777-83 CrossRef Medline 